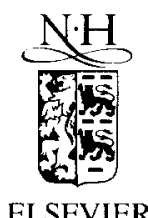

ELSEVIER

\title{
Electrical and magnetic properties of $\mathrm{RCu}$ $(\mathrm{R}=\mathrm{La}, \mathrm{Ce}, \mathrm{Pr}$, and $\mathrm{Nd})$ systems
}

\author{
J.W. Chen \\ Departent of Physics, National Taiwan Unicersity, Taipei, Taiwan
}

Received 3 March 1994; in revised form 8 April 1994

\begin{abstract}
We have investigated the transport and magnetic properties of the $\mathrm{RCu}(\mathrm{R}=\mathrm{La}, \mathrm{Ce}, \operatorname{Pr}$ and $\mathrm{Nd})$ systems by means of the ac electrical resistivity $\rho$ and dc magnetization $\chi$ measurements. For LaCu, bulk superconductivity is observed with a superconducting transition temperature of $6.15 \mathrm{~K}$ as inferred from a sudden drop to zero value in the $\rho(T)$ curve and the diamagnetic behavior in the $\chi(T)$ curve. The occurrence of a peak in the $\chi(T)$ curve and a drop off in the $\rho(T)$ curve indicate that antiferromagnetic transition occurs in $\mathrm{CeCu}$ and $\mathrm{NdCu}$ with transition temperatures $T_{\mathrm{N}}$ of 3.72 and $10 \mathrm{~K}$, respectively. A magnetization study reveals that an antiferromagnetic transition occurs at $T_{\mathrm{N}} \approx 13 \mathrm{~K}$ for $\mathrm{PrCu}$.
\end{abstract}

\section{Introduction}

During the past few decades there have been a lot of research activities on the $\mathrm{R}-\mathrm{Cu}(\mathrm{R}=$ rare earth ions) compounds because of their interesting properties. Among the large variety of $\mathrm{R}-\mathrm{Cu}$ systems, $\mathrm{RCu}$ have been reported to crystallize in orthorhombic FeB-type or cubic CsCl-type structure depending on the $\mathrm{R}$ ions [1-3]. For $\mathrm{R}=\mathrm{La}$, $\mathrm{Ce}, \mathrm{Pr}, \mathrm{Nd}$, and $\mathrm{Yb}, \mathrm{RCu}$ is of the FeB-type structure. Ac susceptibility study of $\mathrm{LaCu}$ indicates that a superconducting transition occurs at $T \sim 5.85 \mathrm{~K}$ [4]. An antiferromagnetic transition was observed in $\mathrm{CeCu}$ with a Néel temperature $T_{\mathrm{N}}$ ranging from 2.7 to $3.6 \mathrm{~K}[2,5]$. Magnetization measurements of $\mathrm{PrCu}$ and $\mathrm{NdCu}$ reveal anomalous characteristics in these compounds [3] and it is possible that $\mathrm{PrCu}$ and $\mathrm{NdCu}$ become magnetically ordered at low temperatures. In this paper, we report ac electrical resistivity and dc magneti- zation studies of the FeB-type $\mathrm{RCu}(\mathrm{R}=\mathrm{La}, \mathrm{Ce}$, $\mathrm{Pr}$, and $\mathrm{Nd}$ ) systems. We found that $\mathrm{NdCu}$ and $\mathrm{PrCu}$ become antiferromagnetic with $T_{\mathrm{N}} \approx 10$ and $13 \mathrm{~K}$, respectively.

\section{Experimental details}

The polycrystalline samples of $\mathrm{RCu}(\mathrm{R}=\mathrm{La}$, $\mathrm{Ce}, \mathrm{Pr}$, and $\mathrm{Nd}$ ) were prepared by arc-melting the high-purity elements ( $\mathrm{R}$ elements: $99.99 \%, \mathrm{Cu}$ : $99.999 \%$ ) together on a water cooled copper hearth in a $\mathrm{Zr}$-gettered argon atmosphere, with no subsequent annealing. Ac electrical resistance $\rho(T)$ measurements on bar-shaped samples were performed in a helium dewar with four-wire technique using a Linear Research Model LR 400 ac resistance bridge operating at a frequency of 16 $\mathrm{Hz}$ with silicon diode and germanium thermometers as temperature sensors. Dc magnetization 
measurements were performed using Quantum Design MPMS SQUID magnetometer from 2 to $300 \mathrm{~K}$ in various applied magnetic fields.

\section{Results and discussion}

The ac electrical resistivity $\rho$ versus temperature $T$ curves for $\mathrm{RCu}(\mathrm{R}=\mathrm{La}, \mathrm{Ce}, \mathrm{Pr}$ and $\mathrm{Nd})$ are plotted in Fig. 1(a) for $0 \mathrm{~K} \leq T \leq 300 \mathrm{~K}$ and in Fig. 1(b) for $0 \mathrm{~K} \leq T \leq 30 \mathrm{~K}$. For $\mathrm{LaCu}$, the superconducting transition occurs in the $\rho(T)$ curve below $6.4 \mathrm{~K}$ with the midpoint transition temperature $T_{\mathrm{c}}(50 \%)=6.15 \mathrm{~K}$ and a transition width $\Delta T_{\mathrm{c}}=0.2 \mathrm{~K}$. Dc magnetization measurement at $H=10 \mathrm{G}$ reveals a diamagnetic transition occurring at the same temperature and indicating bulk superconductivity in this sample.
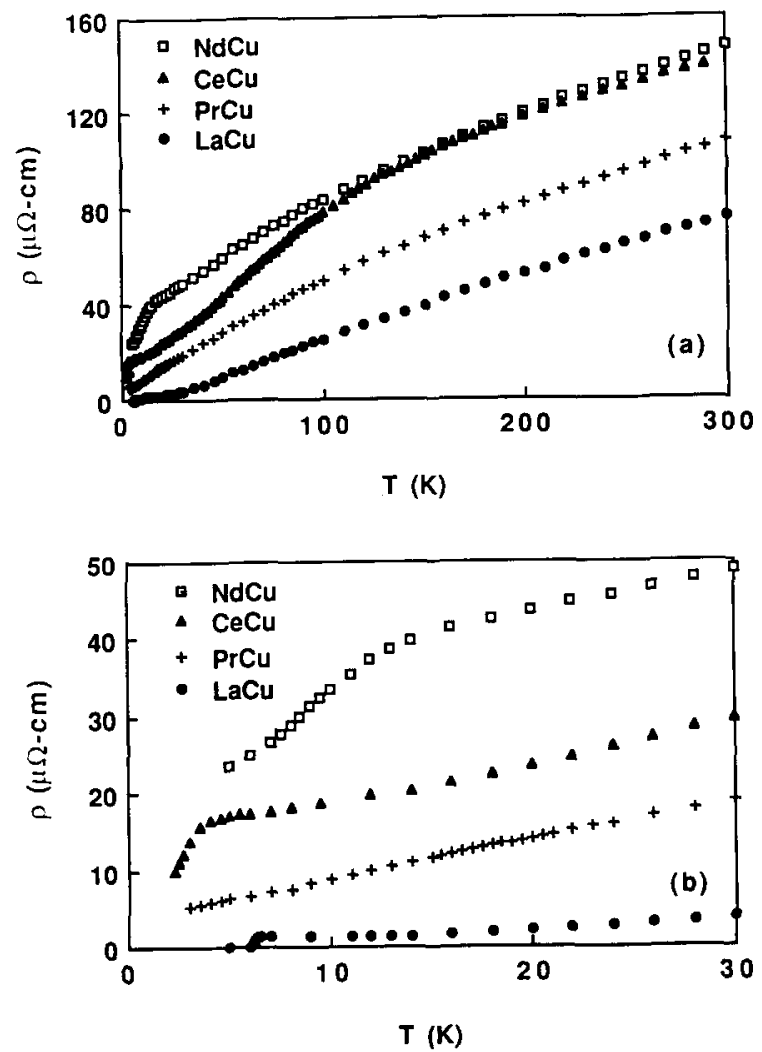

Fig. 1. Electrical resistivity as a function of temperature for $\mathrm{RCu}(\mathrm{R}=\mathrm{La}, \mathrm{Ce}, \mathrm{Pr}$ and $\mathrm{Nd})$ compounds with (a) $0 \mathrm{~K} \leq T \leq$ $300 \mathrm{~K}$ and (b) $0 \mathrm{~K} \leq T \leq 30 \mathrm{~K}$.
Above $T_{\mathrm{c}}$, the $\rho(T)$ curve exhibits typical characteristics of common metals and can be expressed as $\rho(T)=\rho_{i}+\rho_{\mathrm{ph}}(T)$ with a residual resistivity $\rho_{\mathrm{i}}=1.33 \mu \Omega-\mathrm{cm}$. The phonon contribution to the resistivity $\rho_{\mathrm{ph}}(T)$ can be fitted by the semi-emperical Block-Grüneisen formula using a Debye temperature $\Theta_{\mathrm{D}}=120 \mathrm{~K}$ and an electron-phonon interaction constant $C \approx 0.25 \mu \Omega-\mathrm{cm} / \mathrm{K}$. The large value of the $\mathrm{RR}$ ratio $(\rho(300 \mathrm{~K}) / \rho(7 \mathrm{~K})=$ $57)$ reflects the high sample quality. The values of $\rho$ at all temperatures $T$ for $\mathrm{RCu}(\mathrm{R}=\mathrm{Ce}, \mathrm{Pr}$, and $\mathrm{Nd}$ ) are larger than those of $\mathrm{LaCu}$ at the corresponding $T$ and reveal the importance of the magnetic scattering contribution. As shown in Fig. 1(b), a rapid drop in the $\rho(T)$ curves occurs at $T \approx 4 \mathrm{~K}$ for $\mathrm{CeCu}$ and $T \approx 12 \mathrm{~K}$ for $\mathrm{NdCu}$. This indicates that magnetic ordering occurs in these compounds below these temperatures. For $\mathrm{PrCu}$, a small change in the slope of the $\rho(T)$ curve is observed at $T \approx 15 \mathrm{~K}$, in consistency with the magnetization data which show an antiferromagnetic transition at the corresponding temperature (see below).

To obtain a better understanding of the magnetic contribution $\rho_{\text {mag }}$ in these compounds, a semilogarithmic plot of $\rho_{\text {mag }}(T)$ plot is displayed in Fig. 2. The values of $\rho_{\text {mag }}$ are extracted from the measured resistivity $\rho(T)$ of the $\mathrm{RCu}(\mathrm{R}=\mathrm{Ce}$, $\operatorname{Pr}$ and $\mathrm{Nd})$ compounds using $\rho(T)=\rho_{\mathrm{LaCu}}(T)+$ $\rho_{\text {mag }}(T)$, where $\rho_{\mathrm{LaCu}}(T)$ is the resistivity of the isostructural nonmagnetic $\mathrm{LaCu}$ sample at the corresponding temperatures. Above the magnetic ordering temperatures, $\rho_{\text {mag }}(T)$ increases monotonically with $T$ for all compounds; in particular, no In $T$ decrease is observed in $\mathrm{CeCu}$.

Fig. 3 shows the dc magnetic susceptibilities $\chi$ as a function of temperature $T$ for $\mathrm{CeCu}, \mathrm{PrCu}$ and $\mathrm{NdCu}$ measured in an applied field $H=5$ $\mathrm{kG}$ for $0 \mathrm{~K} \leq T \leq 300 \mathrm{~K}$. The magnetic susceptibility for $\mathrm{CeCu}$ exhibits a peak at $T=3.72 \mathrm{~K}$ which indicates the occurrence of antiferromagnetic transition in this compound. While for $T>$ $100 \mathrm{~K}, \chi$ follows a Curie-Weiss behavior $\chi=$ $C /\left(T-\Theta_{\mathrm{p}}\right)$, with $\Theta_{\mathrm{p}}=-42 \mathrm{~K}$ and the Curie constant $C=N \mu_{\text {eff }}^{2} / 3 k_{\mathrm{B}}$ corresponds to an effective moment $\mu_{\text {eff }}=2.62 \mu_{\mathrm{B}}$ per $\mathrm{Ce}$ ion. This is shown in Fig. 4, where $\chi^{-1}$ versus $T$ data for $\mathrm{CeCu}, \mathrm{PrCu}$ and $\mathrm{NdCu}$ are plotted. The obtained 


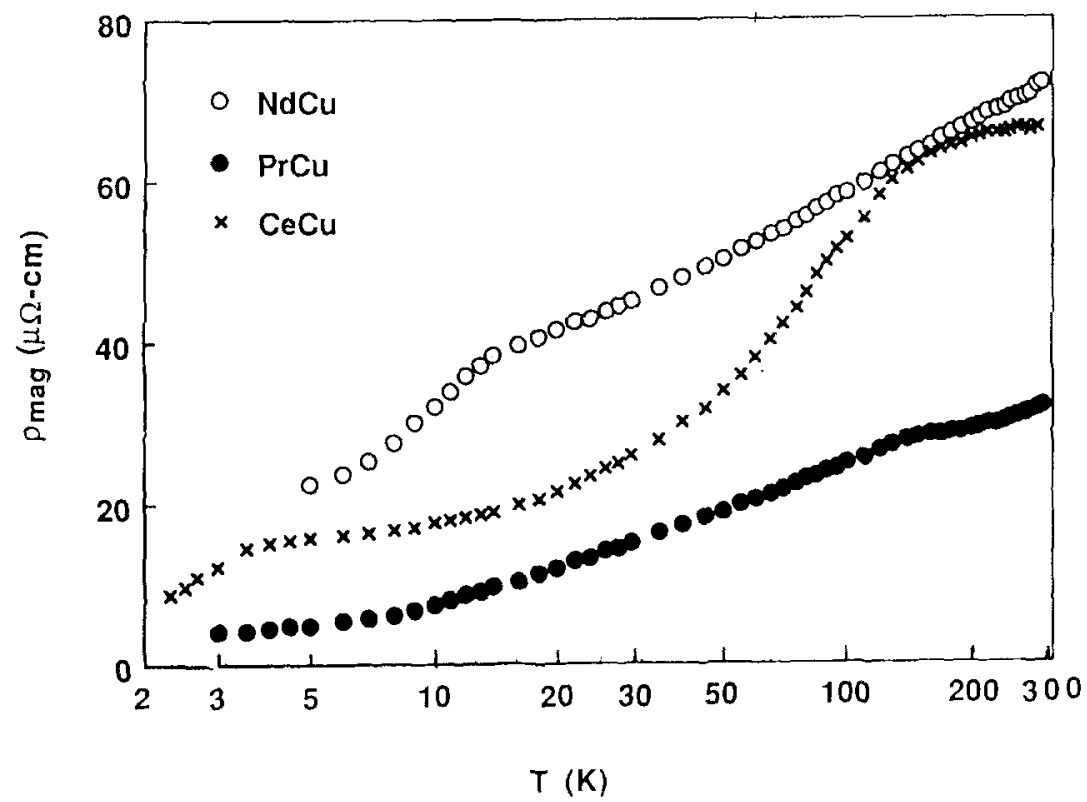

Fig. 2. Magnetic contribution of the resistivity $\rho_{\text {mag }}$ as a function of temperature for $\mathrm{RCu}(\mathrm{R}=\mathrm{Ce}, \operatorname{Pr}$ and $\mathrm{Nd})$ compounds.

value of $T_{\mathrm{N}}$ is consistent with the temperature at which a drop off in the $\rho(T)$ curve occurs for $\mathrm{CeCu}$ and is higher than the values reported previously $[2,5]$.

For $\mathrm{NdCu}, \chi(T)$ also exhibits a peak at $T=10$ $\mathrm{K}$. The peak position is close to the temperature at which a drop in the $\rho(T)$ curve is observed and indicates that antiferromagnetic transition occurs in $\mathrm{NdCu}$ with $T_{\mathrm{N}}=10 \mathrm{~K}$. Above $\sim 150 \mathrm{~K}$, the $\chi(T)$ curve also follows a Curie-Weiss behavior with a value of effective moment $\mu_{\text {eff }}=3.7 \mu_{\mathrm{B}}$ and a Curie-Weiss paramagnetic intercept $\Theta_{p}=-15$ $\mathrm{K}$. The obtained value of $\mu_{\text {eff }}$ is close to the value of the moment $\left(\mu_{\mathrm{eff}}=3.62 \mu_{\mathrm{B}}\right.$ ) for the $\mathrm{Nd}^{3+}$ ions.

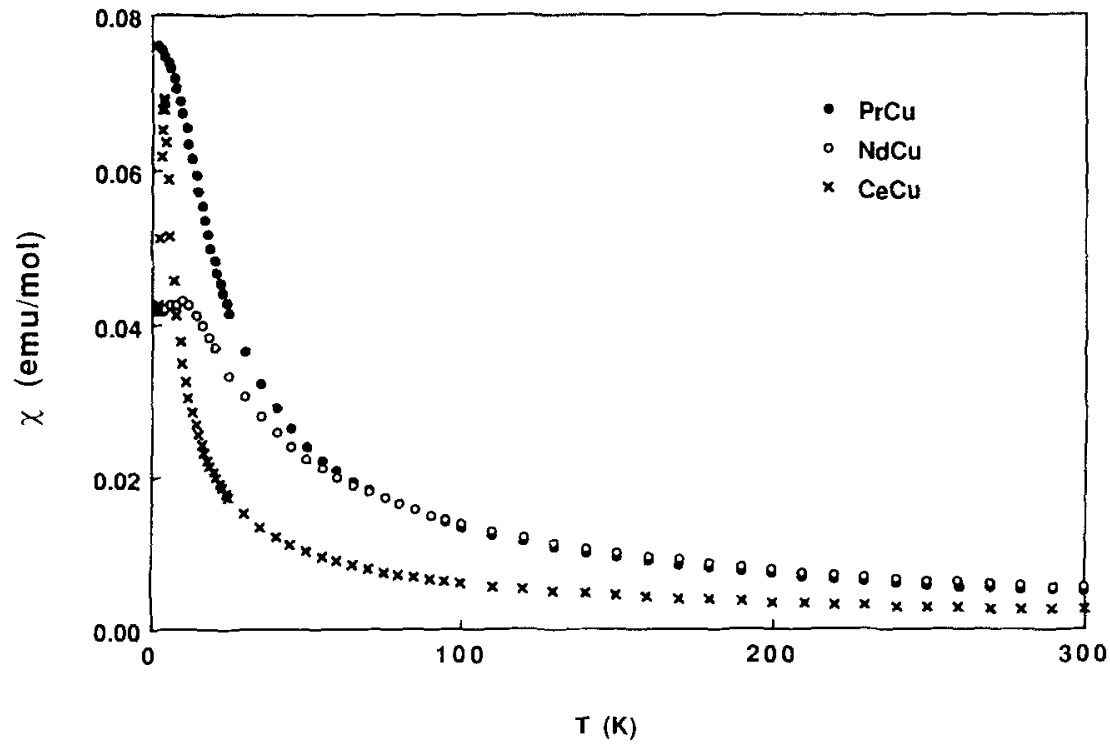

Fig. 3. Magnetic susceptibility as a function of temperature for $\mathrm{RCu}(\mathrm{R}=\mathrm{Ce}, \operatorname{Pr}$ and $\mathrm{Nd})$. The applied magnetic field $H=5 \mathrm{kG}$. 


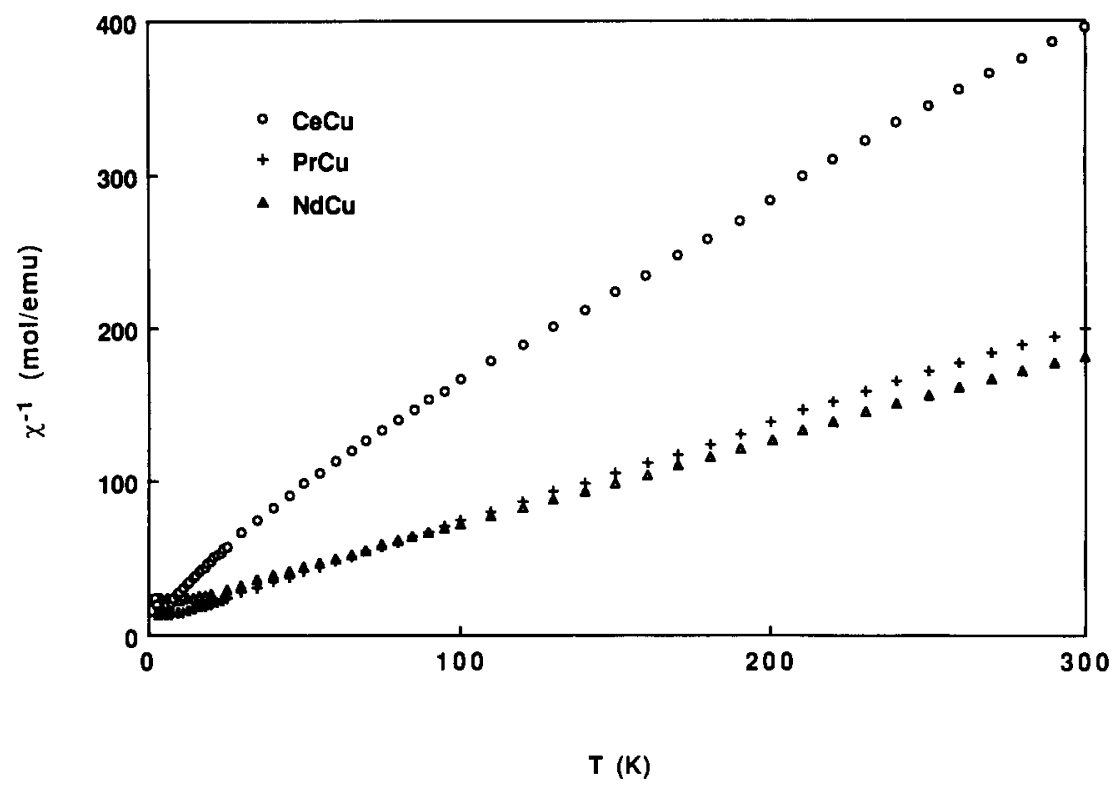

Fig. 4. The inverse susceptibility as a function of temperature for $\mathrm{RCu}(\mathrm{R}=\mathrm{Ce}, \mathrm{Pr}$ and $\mathrm{Nd})$ compounds.

The magnetic susceptibility curve for $\mathrm{PrCu}$ reveals an antiferromagnetic-type transition at $T \approx$ $13 \mathrm{~K}$, which is close to the temperature that a change in the $\rho(T)$ slope was observed. The susceptibility curve $\chi(T)$ can be described with the Curie-Weiss law for $T>160 \mathrm{~K}$ with $\mu_{\text {eff }}=3.52 \mu_{\mathrm{B}}$

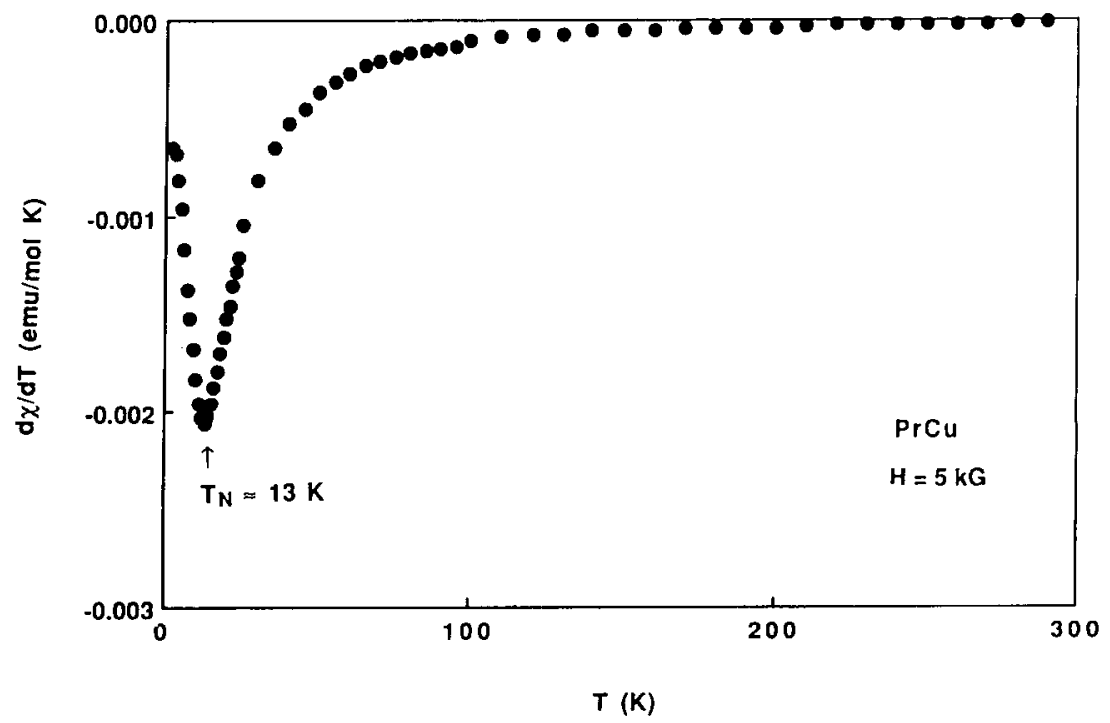

Fig. 5. An antiferromagnetic ordering occurs near $13 \mathrm{~K}$ as reflected by the minimum in the $\mathrm{d} \chi / \mathrm{d} T$ versus $T$ curve in an applied field of $5 \mathrm{kG}$. 
and $\Theta_{p}=-13 \mathrm{~K}$. The value of $\Theta_{\mathrm{p}}$ is close to the observed value of $T_{\mathrm{N}}$ as defined through the inflection point in the $\chi(T)$ curve or the minimum $(T=13 \mathrm{~K})$ in the $\mathrm{d} \chi / \mathrm{d} T$ curve (Fig. 5).

In $\mathrm{CeCu}$, the obtained value of $\mu_{\text {eff }}$ is close to that of the corresponding $\mathrm{Ce}^{3+}$ ions, together with the absence of the $\rho \propto-\ln T$ region in the $\rho_{\text {mag }}(T)$ curve reveals that above the magnetic transition temperature the Kondo interaction plays only a minor role in this system.

In conclusion, we have performed ac electrical resistivity and $\mathrm{dc}$ magnetization studies of the $\mathrm{RCu}(\mathrm{R}=\mathrm{La}, \mathrm{Ce}, \mathrm{Pr}$ and $\mathrm{Nd})$ systems. In addition to reconfirming the existence of a superconducting transition in $\mathrm{LaCu}$ and an antiferromagnetic ordering in $\mathrm{CeCu}$, we have found that $\mathrm{NdCu}$ and $\mathrm{PrCu}$ become antiferromagnetic with $T_{\mathrm{N}} \approx 10$ and $13 \mathrm{~K}$, respectively.

\section{Acknowledgment}

This work was supported by the R.O.C. National Science Council under grant No. NSC 810208-M002-045.

\section{References}

[1] A.C. Larson and D.T. Cromer, Acta. Crystallogr. 14 (1961) 545 .

[2] R.E. Walline and W.E. Wallace, J. Chem. Phys. 42 (1965) 604.

[3] A. Iandelli and A. Palenzona, J. Less-Common Metals 25 (1971) 333.

[4] T.F. Smith and H.L. Luo, J. Phys. Chem. Solids 28 (1967) 569.

[5] E. Bauer, Adv. Phys. 40 (1991) 417. 